\section{Generic $z$-domain discrete-time transfer function estimation for ultra-wideband systems}

\section{R.A. Riaz, M.F.U. Butt, S. Chen and L. Hanzo}

Generic $z$-domain discrete-time transfer function estimation is proposed for ultra-wideband channels, which requires no channel sounding sequence transmission and hence constitutes a blind technique. This is achieved by estimating the channel impulse response with the aid of the information signalling pulses and then equalising the effects of the channel by the corresponding inverse system.

Introduction: Ultra-wideband (UWB) communication systems are characterised by distinct clusters of dense multipath faded received components. In [1], channel estimation techniques were proposed for assisting the receiver in recovering the transmitted UWB signal and hypothesising that an inverse system may be found for signal recovery. Hence the novel contribution of this Letter is that this complete inverse system is indeed found and the corresponding recursive equation is provided for channel estimation. The system does not require any extra channel sounding sequence for the recovery of the received channelcontaminated UWB pulses and so avoids any channel-soundingrelated throughput reduction and therefore may be viewed as a blind technique.

UWB channel model: The statistical channel model specified by the IEEE 802.15 standard is employed for link-budget design. The corresponding wide sense stationary uncorrelated scattering (WSSUS) based channel impulse response (CIR) exhibiting clusters of similar delay paths may be modelled as [2]:

$$
h_{j}(t)=\Theta_{j} \sum_{l=1}^{L} \sum_{w=1}^{W} \beta_{w, l}^{j} \delta\left(t-T_{l}^{j}-\tau_{w, l}^{j}\right)
$$

where $h_{j}(t)$ is the $j$ th CIR realisation, $\beta_{w, l}$ is the $w$ th ray-magnitude of the $l$ th CIR-tap-cluster, while $T_{l}$ is the delay of the $l$ th cluster. Moreover, $\tau_{w, l}$ is the delay of the $w$ th ray relative to the $l$ th cluster's arrival time $T_{l}$. Finally, $\Theta_{j}$ represents the magnitudes of the corresponding cluster components. When assuming perfect symbol synchronisation, (1) may be simplified to:

$$
h(t)=\sum_{l=1}^{L} \beta_{l} \delta\left(t-\tau_{l}\right)
$$

because as stated in [1], perfect symbol synchronisation removes the effects of cluster-overlapping. Furthermore, it was validated by Kolmogorov-Smirnov distribution-fitting to measurements at a significance level of $1 \%$ that the fading amplitudes can be modelled as Nakagami- $m$ distributed [2].

UWB channel estimation: The UWB pulse transmission regime based upon prolate spheroidal wave functions (PSWFs) [3] is employed for transmitting the UWB-PSWF pulse $\phi_{p}(t)$ of width $T, t \in[\tau, \tau+T]$ over the UWB channel characterised by (2). The received signal $r_{p}(t)$ is given by

$$
r_{p}(t)=\phi_{p}(t) * h(t)+n(t)
$$

where $*$ represents the convolution operation, and $n(t)$ is the additive white Gaussian noise (AWGN) having a zero mean and a doublesided power spectral density of $N(s)$. Moreover, when using the orthonormal basis of the Paley-Wiener space [4] for PSWFs, (2) can be expressed as

$$
h(t)=\sum_{n=0}^{\infty} a_{n} \phi_{n}(t)
$$

The $s$-transform of (4) becomes

$$
H(s)=\sum_{q=0}^{\infty} a_{q} \xi_{q} \Phi_{q}(s)
$$

where $\xi_{q}$ represents the specific fraction of energy in the interval $q=$ $0 \ldots \infty$. Taking the $s$-transform of (2) and equating it with (5) we obtain the value for $\beta_{l}$ with UWB-PSWF pulse duration and energy $T$ and $E$, respectively [1]:

$$
\beta_{l}=\sum_{m=0}^{\infty} \sqrt{\frac{E \xi_{m}}{T}} \xi_{m} \phi_{m}\left(\tau_{l}\right)
$$

Substituting (6) into the $s$-transformed version of (2) yields

$$
H(s)=\sum_{l=1}^{L} \sum_{m=0}^{\infty} \sqrt{\frac{E \xi_{m}}{T}} \xi_{m} \phi_{m}\left(\tau_{l}\right) e^{-s \tau_{l}}
$$

The Tustin transformation (TT) $\left\{z \rightarrow e^{s T d}\right\}$ is a conformal mapping employing the sampling time $T_{d}$, in order to convert the $s$-domain channel transfer function (CHTF) $\{H(s)\}$ to the $z$-domain $\{H(z)\}$. Using the following approximation of the TT

$$
\begin{aligned}
s= & \frac{1}{T_{d}} \ln (z)=\frac{2}{T_{d}}\left[\frac{z-1}{z+1}+\frac{1}{3}\left(\frac{z-1}{z+1}\right)^{3}+\cdots\right] \\
& \simeq \frac{2}{T_{d}} \frac{1-z^{-1}}{1+z^{-1}}
\end{aligned}
$$

and substituting (8) into (7) to convert $H(s)$ to $H(z)$ yields

$$
\begin{aligned}
H(z) & =\left.H(s)\right|_{s=\frac{2}{T} \frac{1-z^{-1}}{1+z^{-1}}} \\
& =\sum_{l=1}^{L} \sum_{m=0}^{\infty} \sqrt{\frac{E \xi m}{T}} \xi_{m} \phi_{m}\left(\tau_{l}\right) e \frac{-2 \tau_{l}}{T_{d}} \frac{1-z^{-1}}{1+z^{-1}}
\end{aligned}
$$

When applying the power series expansion of

$$
\frac{e^{\frac{-2 \tau_{l 1-z^{-1}}}{T_{d} 1+z^{-1}}}=\sum_{n=0}^{\infty}\left(\frac{-2 \tau_{l}}{T_{d}} \frac{l-z^{-1}}{1+z^{-1}}\right)^{n}}{n} \simeq 1-\frac{2 \tau_{l}}{T_{d}} \frac{1-z^{-1}}{1+z^{-1}}
$$

and exploiting that $\tau_{l}$ is in the range of nanoseconds, the higher-order components of the power series in (10) can be neglected. Since $\tau_{l}$ is in range of nanoseconds for different range channel models as explained in [2], (10) holds for any value of $\tau_{l}$ between 3-7 ns. Then substituting (10) into (9) yields a general expression for the UWB-channel's $z$ domain discrete-time transfer function (DTTF) having $L$ number of CIR-tap clusters as follows:

$$
H(z)=\sum_{l=1}^{L} \sum_{m=0}^{\infty} \sqrt{\frac{E \xi_{m}}{T}} \xi_{m} \phi_{m}\left(\tau_{l}\right)\left(1-\frac{2 \tau_{l}}{T_{d}} \frac{1-z^{-1}}{1+z^{-1}}\right)
$$

where $\xi_{m}$ is a statistically independent positive random variable having a Nakagami- $m$ probability density function (PDF) with unity fading parameter $m$ for rays within a cluster given by

$$
p\left(\xi_{m}\right)=\left(\frac{2 \xi_{m}}{\overline{\xi_{m}^{2}}}\right) e^{\left(-\xi_{m}^{2} / \xi_{m}^{2}\right)}
$$

Application example: We demonstrate the accuracy of our model using an $L=2$-cluster scenario, where each cluster obey a Nakagami- $m$ distribution with fading parameter of $m=1$ for $\xi_{m}$ in (12). After a few algebraic steps we arrive from (11) at the DTTF:

$$
H_{(L=2, m)}^{*}(z)=\Psi^{\dagger}\left(\frac{1-z^{-1}}{1+z^{-1}}\right)
$$

where

$$
\begin{aligned}
\Psi^{\dagger}= & {\left[( \sqrt { \frac { E \xi _ { m } } { T } } \xi _ { m } ) \left\{\left(\phi_{m}\left(\tau_{1}\right)+\phi_{m}\left(\tau_{2}\right)\right)\right.\right.} \\
& \left.\left.-\left(\frac{2}{T_{d}}\left(\tau_{1 \phi_{m}}\left(\tau_{1}\right)+\tau_{2 \phi_{m}}\left(\tau_{2}\right)\right)\right)\right\}\right]
\end{aligned}
$$

Upon taking the inverse $z$-transform of (13) over the stable region of convergence, the resultant recursive equation can be expressed as

$$
h_{(L=2, m)}^{*}(n)=\Psi^{\dagger} 1^{n} u[-n-1]-\Psi^{\dagger} 1^{n} u[-n-1] \delta[n-1]
$$


Finally, upon substituting $\Psi^{\dagger}$ from (14) into (15), the resultant twocluster Nakagami- $m$ distributed CIR model becomes:

$$
\begin{aligned}
h_{(L=2, m)}^{*}(n)= & {\left[( \sqrt { \frac { E \xi _ { m } } { T } } \xi _ { m } ) \left\{\left(\phi_{m}\left(\tau_{l}\right)+\phi_{m}\left(\tau_{2}\right)\right)\right.\right.} \\
& \left.\left.-\left(\frac{2}{T_{d}}\left(\tau_{1 \phi_{m}}\left(\tau_{1}\right)+\tau_{2 \phi_{m}}\left(\tau_{2}\right)\right)\right)\right\}\right] 1^{n} u[-n-1] \\
& -\left[( \sqrt { \frac { E \xi _ { m } } { T } } \xi _ { m } ) \left\{\left(\phi_{m}\left(\tau_{1}\right)+\phi_{m}\left(\tau_{2}\right)\right)\right.\right. \\
& \left.\left.-\left(\frac{2}{T_{d}}\left(\tau_{1 \phi m}\left(\tau_{1}\right)+\tau_{2 \phi m}\left(\tau_{2}\right)\right)\right)\right\}\right] 1^{n} u[-n-1] \delta[n-1]
\end{aligned}
$$

The CIR of (13) was restricted to retain only those multipath components, which are less than $12 \mathrm{~dB}$ below the maximum multipath component. The UWB-PSWF pulse duration was $0.15 \mathrm{~ns}$, which was transmitted every $120 \mathrm{~ns}$ and the channel's inverse system was estimated for each new pulse. Finally, a threshold-detector is used to recover the transmitted data. The BER against signal-to-noise ratio (SNR) performance is shown in Fig. 1, demonstrating that using $W=15$ taps in the proposed model approaches the full complexity performance-evaluation curve of (19) in [1].

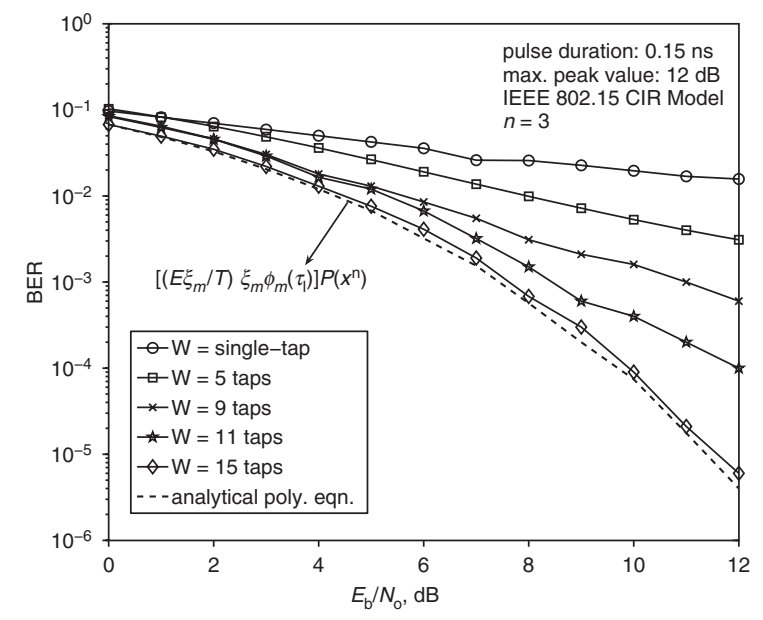

Fig. 1 Accuracy of proposed z-domain DTTF estimation of (16) for $L=2$ and $W=1,5,9,11,15$ CIR taps
Conclusion: The generic $z$-domain DTTF estimation proposed for UWB systems facilitates accurate performance evaluation and requires no channel sounding sequence.

(C) The Institution of Engineering and Technology 2008 30 August 2008

Electronics Letters online no: 20082536

doi: 10.1049/el:20082536

R.A. Riaz, M.F.U. Butt, S. Chen and L. Hanzo (School of ECS, University of Southampton, SO17 1BJ, United Kingdom)

E-mail: 1h@ecs.soton.ac.uk

\section{References}

1 Dilmaghani, R., Ghavami, M., and Aghvami, H.: 'Channel estimation technique for ultra-wideband pulse transmitters', Electron. Lett., 2004, 40, pp. $1348-1350$

2 Molisch, A.F., Cassioli, D., Chong, C.C., Emami, S., Fort, A., Kannan, B., Karedal, J., Kunisch, J., Schantz, H.G., Siwiak, K., and Win, M.Z.: 'A comprehensive standardized model for ultrawideband propagation channels', IEEE Trans. Antennas and Propag., 2006, 54, pp. 3151-3166

3 Parr, B., Byunglok, C., Wallace, K., and Ding, Z.: "A novel ultrawideband pulse design algorithm', IEEE Commun. Lett., 2003, 7, pp. $219-221$

4 Slepian, D.: 'Prolate spheroidal wave functions, Fourier analysis, and uncertainty. V - The discrete case', Bell Syst. Tech. J., 1978, 57, pp. $1371-1430$ 